A N N A L E S Annales de Bretagne et des Pays de l'Ouest

\title{
Trésors enluminés de la Bretagne au Moyen Âge
}

\section{Franck Mercier}

\section{OpenEdition}

Journals

Édition électronique

URL : https://journals.openedition.org/abpo/4861

DOI : $10.4000 / a b p o .4861$

ISBN : 978-2-7535-8021-3

ISSN : 2108-6443

Éditeur

Presses universitaires de Rennes

Édition imprimée

Date de publication : 20 décembre 2019

Pagination : 224-226

ISBN : 978-2-7535-8019-0

ISSN : 0399-0826

Référence électronique

Franck Mercier, "Trésors enluminés de la Bretagne au Moyen Âge », Annales de Bretagne et des Pays de I'Ouest [En ligne], 126-4 | 2019, mis en ligne le 22 janvier 2020, consulté le 05 janvier 2023. URL : http:// journals.openedition.org/abpo/4861; DOI : https://doi.org/10.4000/abpo.4861 
VInCENT-LAnglois, Stéphanie, Trésors enluminés de la Bretagne au Moyen Âge, Éditions Ouest-France, Rennes, 2018, 381 p.

L'ouvrage de Stéphanie Vincent-Langlois est d'abord un " beau livre " qui se conçoit comme une invitation à découvrir les " trésors " cachés dans les manuscrits à peinture de la période médiévale au service d'une certaine idée de la Bretagne. L'ambition est clairement de toucher le grand public qu'il s'agit de séduire, par la qualité et la richesse de l'illustration, autant que d'instruire, à travers une présentation pédagogique du Moyen Âge " breton " en général et des enluminures en particulier.

Conformément au projet initial, le livre prend essentiellement appui sur un ensemble de quatre manuscrits de luxe liés d'une façon ou d'une autre, et selon une définition sans doute plus " patrimoniale " qu'historique, à l'histoire de la Bretagne : la Compilation des chroniques et des histoires de Bretagne de Pierre Le Baud (Paris, BnF, Ms. fr. 8266); les Grandes Heures d'Anne de Bretagne (BnF, Ms. Lat. 9474); le Livre d'Heures de Montauban (Rennes, Bibliothèque de Rennes Métropole, Ms. 1834 et BnF, Ms. fr. 18026) ainsi que les Heures de Catherine de Rohan et de Françoise de Dinant (Rennes, BRM, Ms. 0034/0034bis/0034ter). On ne s'étonnera pas de voir figurer en tête de cette liste le splendide et célèbre manuscrit de la Compilation des chroniques et des histoires de Bretagne. Réalisé à la fin du xve siècle pour le compte de Jean de Derval, il constitue précisément une tentative pour élaborer une histoire du duché de Bretagne en l'enracinant le plus loin possible dans le temps, comme pour mieux l'autonomiser face au royaume de France. Ce n'est assurément pas un hasard si la fameuse scène de dédicace (commentée p. 38) sert de couverture à l'ouvrage des éditions Ouest France. Si nombre d'enluminures tirées de ce manuscrit sont exploitées tout au long de l'étude à des fins illustratives, il se pourrait aussi que la conception même du livre de Stéphanie Vincent-Langlois, dans la perspective historique qui est la sienne, doive quelque-chose d'essentiel, même à son insu, au programme idéologique qui sous-tendait la Compilation de Pierre Le Baud : affirmer l'existence éternelle d'une Bretagne (ducale) indépendante du royaume de France.

Telle n'est pas cependant l'intention explicite de ce livre qui se veut d'abord un superbe album d'images en même temps qu'un hommage conscient et appuyé à son prédécesseur, le codex enluminé médiéval et plus particulièrement le livre d'heures du xve siècle, dont l'ouvrage présent tend d'ailleurs à imiter la forme matérielle. Ce souci mimétique se traduit dans le format assez compact et maniable du volume, dans les mises en page et jusque dans les choix typographiques, comme le parti insolite de scander le texte par des pieds-de-mouche de couleur rouge et bleue. L'ouvrage est un régal pour les yeux : l'abondance des détails en gros plan fait aisément oublier sa taille relativement réduite $(17 \times 21,5 \mathrm{~cm})$. La qualité des reproductions, à une exception près (p. 127), est remarquable et donne à voir des détails peu visibles sur d'autres éditions. On retrouve bien sûr à l'intérieur de l'ouvrage les fleurons escomptés de l'imagerie attachée à la Bretagne ducale (comme celles extraites de la Compilation des chroniques de Bretagne, avec notamment l'image de la forteresse de Châteaugiron longuement commentée p. 256-260, ou encore du Grand Armorial équestre de la Toison d'or avec son duc de Bretagne en posture équestre entièrement revêtu de l'hermine héraldique) mais aussi de véritables raretés qui 
renforcent l'intérêt de cette publication (le duc Jean V en armure, p. 294, ou encore la bataille de saint Aubin du Cormier, p. 312).

La présentation générale se veut résolument pédagogique : après une introduction destinée à présenter les rudiments de la culture livresque au Moyen Âge, l'ouvrage propose une vue cavalière de l'histoire de la Bretagne, depuis le "temps des Romains " jusqu'à la fin de l'autonomie ducale et la "réunification à la France ", en passant par une évocation des vikings, de la guerre de succession de Bretagne (1341-1364), de la Guerre de Cent ans, etc. Assez logiquement compte-tenu de l'origine principale des enluminures, c'est surtout la Bretagne ducale à la fin du Moyen Âge qui se taille la part du lion ou plutôt, serait-on tenté de dire, de l'hermine, tant ce motif héraldique, porteur d'une aspiration à la souveraineté, devient récurrent et présent visuellement à partir du XIV siècle. Entrecoupés de courtes séquences thématiques (consacrées à la mort, aux saints bretons, etc.), ce survol historique est surtout le prétexte ou le support à une foisonnante illustration établie à partir d'une sélection d'enluminures, pour la plupart extraites de manuscrits à peinture tardifs, à commencer par nos " quatre trésors enluminés de Bretagne ". L'ensemble crée un effet kaléidoscopique qui donne à l'ouvrage l'allure de " très riches heures " bretonnes adaptées à l'air du temps.

Si la relation entre texte et enluminure est finalement peu explorée, chaque illustration fait l'objet d'un commentaire précis qui éclaire l'événement représenté sans omettre l'examen de l'image elle-même. Dans le droit fil de l'iconographie de François Garnier dont l'auteur se réclame, l'image est toujours appréhendée comme un objet à décrypter et à " lire " selon les codes d'une grammaire avant tout symbolique de l'image. L'intention pédagogique perce toujours sous le discours savant : émaillée de brèves mises au point sur la société ou la civilisation médiévale, l'analyse des images est le plus souvent claire et pertinente et joue parfaitement son rôle explicatif (dans l'identification des personnages, des saints, des attributs symboliques, des signes héraldiques, etc.).

On peut toutefois regretter que le commentaire cède encore trop facilement à certains clichés historiographiques que ne sauraient toujours justifier la seule licence pédagogique ou le souci d'atteindre le grand public. Si l'on peut ainsi admettre l'usage entre guillemets du mot " carte " pour désigner l'une des premières représentations connues de la péninsule bretonne (p. 66), on déplorera certaines schématisations convenues et depuis longtemps contestées par la science historique. Ainsi, le fait de réduire la transition du Moyen Âge à la "Renaissance " (un peu trop vite identifiée à une période historique) au passage de la représentation " symbolique " au " réalisme " apparaît très simplificateur. Récurrente dans l'ouvrage, cette référence discutable au "réalisme ", par opposition au " symbolisme " qui serait inhérent aux " mentalités médiévales ", apporte plus de confusion que de clarté lorsqu'il s'agit de penser le rapport des images au réel. Parler ainsi sans nuances de " portrait " ou de " paysage armoricain ", même à propos d'enluminures tardives, apparaît très contestable. De même, on ne peut laisser dire que «la guerre, depuis le milieu du XIV ${ }^{\mathrm{e}}$ siècle, n'est plus à la lutte chevaleresque en bataille rangée, respectueuse du code d'honneur " (p. 259), dans la mesure où elle ne l'a jamais été à l'époque féodale comme le rappellent, entre autres, les travaux déjà anciens de 
Georges Duby. À visée surtout pédagogique et patrimoniale, le propos ne s'embarrasse guère non plus de subtilités historiographiques. Les duchés de Bretagne et le royaume de France sont ainsi des entités politiques appréhendées de manière atemporelle sur le modèle de l'état-nation : les " bretons " sont vaincus par les " français " à la bataille de saint Aubin du Cormier (p. 313) - alors qu'on sait que les seigneurs " bretons " étaient au moins aussi nombreux dans le camp royal que dans le camp ducal - et la Bretagne ducale est l'objet d'une "réunification à la France " (p. 364) - qui supposerait donc une pleine unification préalable. Ces quelques réserves ou critiques demeurent cependant secondaires par rapport à l'ambition véritable de l'ouvrage qui consiste bien d'abord à présenter, de la manière la plus valorisante, généreuse et pédagogique possible, les incontestables richesses ou " trésors " des livres enluminés légués par le Moyen Âge. Si l'intention était de montrer la Bretagne médiévale (en fait surtout ducale) sous ses plus beaux atours et telle que le grand public ne l'avait encore jamais vue, alors le pari des Éditions Ouest-France et de Stéphanie Vincent-Langlois est assurément réussi.

Franck MERCIER

ABELARD, Karine (édition établie et commentée par), Compillation des cronicques et ystoires des Bretons de Pierre LE BAUD, Rennes, Presses Universitaires de Rennes et Société d'Histoire et d'Archéologie de Bretagne, coll. « Sources de la Bretagne médiévale ", 2018, 630 p.

Un nouveau volume vient enrichir la remarquable collection des « Sources de la Bretagne médiévale " publiée par les PUR et la Société d'histoire et d'archéologie de Bretagne. Après avoir consacré plusieurs publications aux documents diplomatiques - actes des ducs, cartulaires de Saint-Melaine ou de Quimperlé - et aux comptes ducaux, elle aborde un nouveau champ, celui des historiens et commence par un auteur de première grandeur, même s'il demeure mal connu, Pierre Le Baud En effet, son œuvre demeura longtemps éclipsée par celles de ses successeurs, Alain Bouchart et Bertrand d'Argentré car elle était manuscrite quand ces derniers bénéficiaient de l'imprimerie. La diffusion en fut donc restreinte et, à ce jour nous ne disposions que d'une version imprimée par d'Hozier au XVII ${ }^{\mathrm{e}}$ siècle et encore dans une autre version. Enfin, Ch. De La Lande de Calan au début du Xxe édita bien la Compilation mais ne put achever le travail. C'est donc, à bien des points de vue, une excellente initiative que de nous donner une version complète et bien établie de la Compillation des cronicque et ystoires des Bretons, le premier essai d'une vaste histoire de Bretagne réalisée à un moment crucial pour le duché. Le Baud a vécu les tribulations de la fin de l'indépendance, et fut un témoin privilégié étant au service de Jean de Derval puis de la duchesse Anne. En même temps, il fut un témoin engagé car il s'intègre complètement dans le mouvement d'intellectuels qui, à la fin du Moyen Âge et après, tinrent un discours destiné à forger ou renforcer un sentiment national dans le duché. 